\title{
Application of Data Architecture Model in Enterprise Management
}

\author{
Song Shi, Yalin Ji \\ Ordnance Engineering College, Shijiazhuang, 050003, China
}

\begin{abstract}
Today is in the era of rapid development of information, data volume of high-speed expansion, it is difficult in the previous system for communication, sharing and integration. In order to integrate data resources, eliminate the "information island", build enterprise development blueprints, people gradually realize the importance of top design. Many enterprises for their own development to establish their own enterprise architecture of the top design, and as its core data architecture model is also reflected in different industries according to different development. This paper mainly studies the data architecture model, expounds the role of data architecture model and its relationship.
\end{abstract}

\section{Introduction}

Data and information is the economic lifeline of the $21 \mathrm{st}$ century. In the information age, the data is considered to be an important corporate asset. Often a company has long accumulated a lot of demand, description, database, data model, procedures and a variety of information technology finished components, but the lack of an "overall design."

This is not to say which information system, database or database management system (DBMS) is wrong or misleading, but said that the current generation of a large number of information technology (Information Technology, IT) components, and the urgent need to establish a " General structure "to its specification, optimization or even standardization.

In the previous data warehouse project, the concept and methodology of the system data model usually focuses on how to design and build a data warehouse rationally, but rather an application system (OLTP system) models design without the need for effective methodological guidance.The application system are different in different periods by different manufacturers in the design and development, and lack of communication between each other, resulting in data scattered and repeated, inconsistent caliber and poor data compatibility. As the data warehouse in the overall enterprise information planning belongs to the downstream system, it can only passively receive data generated by the application system, data warehouse. Due to inconsistent caliber, poor compatibility, it brings great difficulties in data integration. Enterprises in a lot of manpower, material and capital to promote information technology, but there are still a lot of "information island" phenomenon.

This paper argues that the success of enterprise information construction depends largely on the rationality of the system model and the consistency of the concept of different systems. The enterprise data model is the core of enterprise information. Through the enterprise data model to define the entire enterprise information system of data standards, and gradually unify the internal data standards. It will also guide the application system data model unified design, and can fundamentally guarantee the data onto the system compatibility and consistency. Enterprise data model can eliminate data fragmentation, inconsistency, and information islanding due to the design and development of the application system. It can promote the integration of various applications within the enterprise and data sharing, and comprehensively enhance the business decision-making, operations management, business development , customer service and other aspects of support capabilities.

\section{Definition of enterprise data architecture model}

\subsection{Basic definition of data architecture model}

Enterprise-level data architecture models, is a method used to define the regular representation of information. By using the information model, we can use different applications to reuse, change, and share the data that is being managed. Enterprise data architecture can help enterprises to eliminate information island, and build a shared, generic, consistent and extensive enterprise data infrastructure platform. ${ }^{[1]}$

The enterprise data model can not be equated with the data warehouse model. The enterprise data model is the perspective of the whole enterprise business, and the whole description of the whole enterprise data (including production data and consumption data). Enterprise-level data model is the business and IT staff to communicate 
the media, but also within the enterprise and external communication.

\subsection{Data model structure}

A reference model for an enterprise's data architecture includes:

- Data element model

- Conceptual data model

- Database logic model

- Database physical model

- A view model that connects physical databases and application information systems

The five data structures above form a core, and around the core of a large number of information technology support can be interconnected, interoperable, and non-redundant. All the information technology products, including data structure reference model of those are drawn in a map inside, you will get as shown in Figure 1.

We need an application information system with a "meta-database" cores. The data model for this "metabase" is highly engineered, and it really supports interrelated, interoperable, and non-redundant. ${ }^{[2]}$

\section{Data architecture reference model}

The data architecture of the enterprise and its accompanying data models have always been seen as important assets. Figure 1 of the preceding article illustrates how important this asset is. This importance is not only reflected in the fact that the data model forms the basis of the corporate strategy, and the data model is the ultimate "intersection" of all information technology products.

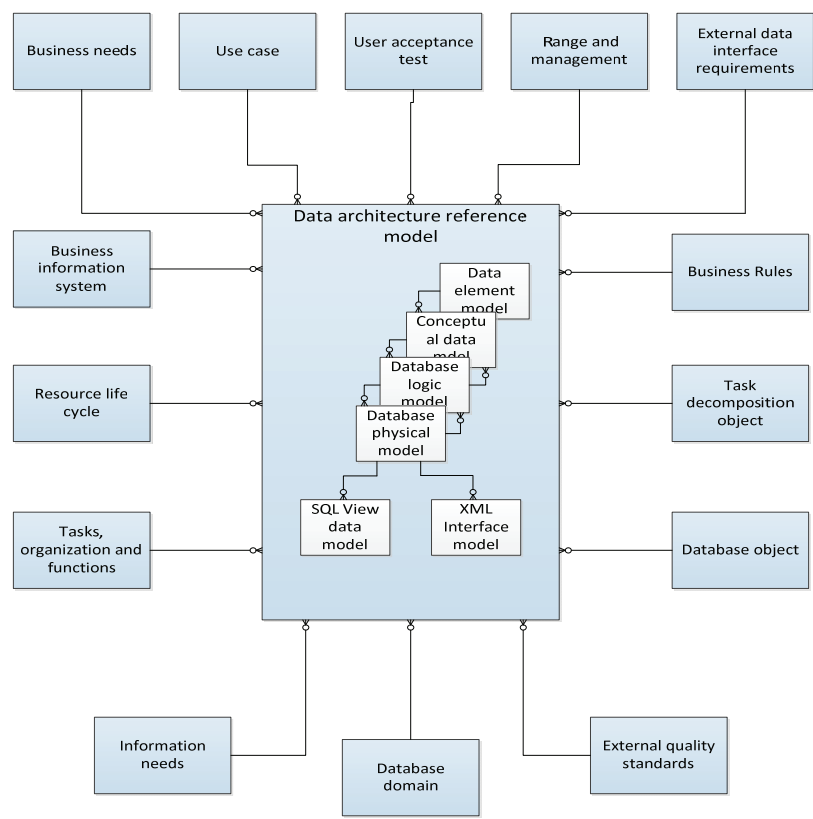

Figure 1. Enterprise architecture product set and data architecture reference model

The separate data models in the data architecture are independent of each other and handle different data separately. These architectures promote model integrity, interoperability, and non-redundancy. In addition, a "more advanced" model can be used as a template for creating "low" models.

Once the reference model of the data architecture has been completed, it can serve as a framework to help understand the scope and interrelationship of the component model. ${ }^{[3]}$

This model is useful for communication between component instances in the data architecture model.

Our understanding of the natural concepts of data, database deployment, and business information systems has evolved over the years, and on this basis, we can see that the data structure reference model ( Figure 2) has six separate models. The six models are:

- Data element model

- Concept data model

- Logical database model

- Physical database model

- SQL view model

- XML document structure model

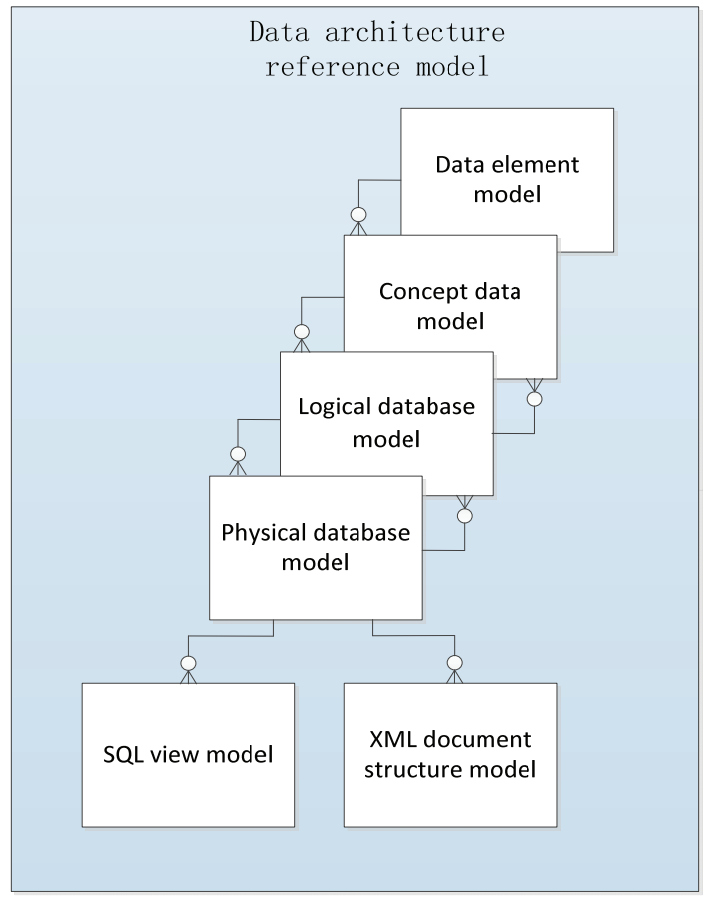

Figure 2. Data architecture reference model

\section{The role of each model in the data architecture reference model}

\subsection{Data element model}

The data element is the essence of the enterprise and is used as the semantic basis of the entity attribute in the conceptual data model. It supports the columns of the database model required by the business and is implemented in the database management system. ${ }^{[4]}$ It is used in the business information system (view Data model), to achieve the enterprise resources required for the database object, and to support the completion of corporate tasks. 


\subsection{Concept database model}

The conceptual data model consists of the subject, the entity, the relationship between the attribute and the entity. Relationships can reflect the interrelationships among multiple subjects. Each data model should correspond to only one concept, such as a person's name, or an address. The conceptual data model can be used as a template when creating a database model (logical data model or physical data model). Each entity attribute should be mapped to its parent data element. Semantics and data usage modifiers can be attributed to each entity attribute. The key components are themes, entities, attributes, and relationships.

The conceptual data model can be expressed as a container, such as a student, a school, an organization, or an address. These containers (such as students or schools) must be described in detail before they can be implemented in one or more different database tables, and eventually become objects that can be operated by a database management system such as Oracle. ${ }^{[5]}$

\subsection{Logical database model}

The logical data model is a data model that is independent of the database of the database management system. The data structure components that make up the model includes: patterns, tables, columns, and the relationship between tables. The relationship is limited to a single schema table. Although each implementation of the database model can correspond to multiple descriptive data models, but each implementation of the database model should only correspond to a problem. Each column of the table should be mapped to a parent attribute. Semantics and data usage modifiers can be attributed to each column. There are many-to-many relationships between the description data model and the implementation of the database model. The key components are patterns, tables, columns, and relationships.

\subsection{Physical database model}

The data model is a data model that is bound to a database of a particular database management system. The data structure components that make up the model include: database management system model, database management system table, database management system column and the relationship between the database management system tables. The database management system relationship is limited to a single schema in the database management system table. Each physical database model can correspond to multiple logical database models. Each column of the database management system should be mapped to a parent column. There is a many-to-many relationship between the logical database model and the physical database model. The key components are database management system model, database management system table, database management system and database management system relationship. ${ }^{[6]}$
If the data model is dependent on the state of a particular database management system and a particular software application performance, the data model is called "physical", and the data model is a physical database model that is bound to the application business information system through a view data model. Operating the database model inside the database management system table is to achieve the database model inside a single table in a single column deployment.

\subsection{View data model}

The view data model represents the interface between the physical database model and the business information system. The column of views and views can be characterized as input / output. ${ }^{[7]}$ In addition, these views can be mapped to each other on a column basis, and the process can be specified to define arbitrary data value conversions.

The view data model is bound to a specific database management system by its definition. The view data model enables an application system to select, apply, or update a database system based on its physical data model, without having to include details of the physical data model in the application system. ${ }^{[8]}$

\section{Conclusion and outlook}

Enterprise architecture is a strategic information asset. It describes the current and expected relationship between an organization's business, mission, management process, and supporting infrastructure. The data architecture is the core of the enterprise architecture. It evolves business or mission into a more efficient, more efficient, and more popular fashion. It enhance the interoperability, and the sharing of information. At the same time, it improve business processes, and promote major decision-making planning.

Many enterprise systems have the same architectural approach, although an organization can have many different genres and practices in the implementation of enterprise data architecture. This demonstrates a variety of technical patterns and designs that are independent of a particular application area, are effective against generating responsive, scalable, flexible, and unified enterprise applications. The development of enterprises can not be separated from the enterprise data architecture, and reasonable data architecture applications may be the future of information society enterprises to survive the key.

\section{References}

1. Hailan Yu. Enterprise architecture. [M]. (2009)

2. Libo Xu. Model Design of Data Warehouse in Book Chain Enterprise. [J]. (2011)

3. Chengliang Lin. Power supply enterprise data model analysis and design

4. Genbao Zhang. Enterprise Informatization. [M]. (1999) 
5. Enterprise architecture.

Www.wiscorp.com/sp/sp08.pdf

6. Jijiang Yang,Wei He,Chunxiao Xing. Construction of e-government general data model [J]. Egovernment .1.(2001)

7. Engineering and Management Information System Planning www.wiscorp.com/sp/sp11.pdf

8. Adamson, Christopher and Michael Venerable. Data Warehouse Desgn Solutions. John Wiley \& Sons, 544(1998) 